\title{
Kinematic Rules for Upper and Lower Arm Contributions to Grasp Orientation
}

\author{
J. J. Marotta, ${ }^{1}$ W. P. Medendorp, ${ }^{1,2}$ and J. D. Crawford ${ }^{\mathbf{1}}$ \\ ${ }^{1}$ Canadian Institutes of Health Research Group for Action and Perception, Department of Psychology, Centre for Vision Research, \\ York University, Toronto, Ontario M3J-1P3, Canada; and ${ }^{2}$ Nijmegen Institute for Cognition and Information, University of Nijmegen, \\ Nijmegen, The Netherlands
}

Submitted 30 April 2003; accepted in final form 18 August 2003

\begin{abstract}
Marotta, J. J., W. P. Medendorp, and J. D. Crawford. Kinematic rules for upper and lower arm contributions to grasp orientation. $J$ Neurophysiol 90: 3816-3827, 2003. First published August 20, 2003; $10.1152 / \mathrm{jn} .00418 .2003$. The purpose of the current study was to investigate the contribution of upper and lower arm torsion to grasp orientation during a reaching and grasping movement. In particular, we examined how the visuomotor system deals with the conflicting demands of coordinating upper and lower arm torsion and maintaining Donders' Law of the upper arm (a behavioral restriction of the axes of arm rotation to a two-dimensional "surface"). In experiment 1 , subjects reached out and grasped a target block that was presented in one of 19 orientations $\left(5^{\circ}\right.$ clockwise increments from horizontal to vertical) at one position in a vertical presentation board. In experiment 2, target blocks were presented in one of three orientations (horizontal, three-quarter, and vertical) at nine different positions in the presentation board. If reach and grasp commands control the proximal and distal arms separately, then one would only expect the lower arm to contribute to grasp orientations and that Donders' Law would hold for the upper arm-independent of grasp orientations. Instead, as the required grasp orientation increased from horizontal to vertical, there was a significant clockwise torsional rotation in the upper arm, which accounted for $9 \%$ of the final vertical grasp orientation, and the lower arm, which accounted for $42 \%$. A linear relationship existed between the torsional rotations of the upper and lower arm, which indicates that the components of the arm rotate in coordination with one another. The location-dependent aspects of upper and lower arm torsion remained invariant, however, yielding consistently shaped Donders' "surfaces" (with different torsional offsets) for different grasp orientations. These observations suggest that the entire arm-hand system contributes to grasp orientation, and therefore, the reach/grasp distinction is not directly reflected in proximal-distal kinematics but is better reflected in the distinction between these coordinated orienting rules and the location-dependent kinematic rules for the upper arm that result in Donders' Law for one given grasp orientation.
\end{abstract}

\section{N T R O D U C T I O N}

Humans are capable of elegantly controlled visually guided reaching and grasping movements. During these movements, there are an "infinite" number of finger and arm postures that will successfully place the hand on a target. The act of grasping an object is the outcome of simultaneous movements at several

Address for reprint requests and other correspondence: J. Marotta, Dept. of Psychology, Behavioural Sciences Building, York Univ., 4700 Keele St., Toronto, Ontario, M3J-1P3 Canada (E-mail: marotta@yorku.ca). joints that transport the arm to the vicinity of the target, bring the orientation of the hand in accord with object tilt, and preshape the fingers into an appropriate grip. Although these components of reaching-grasping movements differ widely in terms of their biomechanics and the details of their neural organization, they must all be coordinated to achieve a stable grasp for holding and manipulating the object (Paulignan et al. 1997). A continuing problem in motor control is to understand the constraints employed by the CNS in controlling a kinematically redundant motor apparatus, like the arm. That is, while the arm has seven degrees of freedom available, six degrees would suffice to specify the position and orientation of its end effector (the hand) in space. The fact that arm movements with the same start and endpoints are made in a consistent, reproducible fashion suggests that there is a reduction is the number of the degrees of freedom. How this reduction is realized is a central theme in the area of motor control. Here, we attempt to integrate two very different solutions to the kinematic redundancy problem, the "parallel visuomotor processing approach" and the "Donders' Law approach".

\section{Parallel visuomotor processing theories of grasping}

One way the brain could deal with the complexity of a reaching and grasping movement is to split the total movement plan into parallel functional modules, each involving a small number of degrees of freedom (Arbib 1981; Arbib et al. 1985; Gentilucci et al. 1991; Jeannerod 1981, 1992; Jeannerod and Biguer 1982; Paulignan et al. 1991a,b; Stelmach et al. 1994). According to this theory, prehension movements directed to visual stimuli can be partitioned into three independent components: the transport of the arm, the orientation of the hand, and the grasp (Arbib 1981; Jeannerod 1988). The transport component involves proximal joints and muscle groups, while the grasping component involves distal joints and muscles (Arbib 1990; Jeannerod 1984; Jeannerod and Decety 1990; Lacquaniti and Soechting 1982; Stelmach et al. 1994). The peak transport velocity of the limb is usually achieved one-third of the way through the total duration of the reach and is followed by a prolonged deceleration phase as the target is approached.

\footnotetext{
The costs of publication of this article were defrayed in part by the payment of page charges. The article must therefore be hereby marked "advertisement" in accordance with 18 U.S.C. Section 1734 solely to indicate this fact.
} 
Within the initial acceleration of the arm transport, the hand's posture and orientation are adapted according to the task. Hand shaping, determined by visual assessment of object dimensions, will also be assumed during the acceleration phase in anticipation of contact (but see Smeets and Brenner 1999 for an alternative view). While the parallel processing theory seems to control different sets of degrees of freedom, the system still must resolve the kinematic redundancy problem, i.e., six task or object coordinates must be mapped onto seven controlled joints.

The parallel visuomotor processing theory has been supported by a large number of psychophysical experiments. Reaching for the same object presented at different locations in space has been shown to influence the kinematics of the transport component of a reach without affecting the grasp (Gentilucci et al. 1991; Jeannerod 1984). Conversely, when objects of different size are presented at a given location, the modification of grip aperture has been shown not to influence the transport component of the movement (Paulignan et al. 1991a; Wallace and Weeks 1988).

Constancy of the arm transport can also be observed when the object to grasp is presented along different orientations in space (Lacquaniti and Soechting 1982; Stelmach et al. 1994). For example, in a task where subjects were required to reach out and grasp a target presented at different orientations, (Lacquaniti and Soechting 1982) found that the motion of the wrist was more variable in timing and duration than that of the shoulder and elbow. They argued that wrist motion is controlled separately from shoulder or elbow motion and that there may not be advantages in having wrist rotation develop synchronously with the motions at the other two joints. It should be noted, however, that subjects in this experiment were forced to move their wrist along a straight line to the target, in the sagittal plane. In fact, this constraint removes one degree of freedom, and as such, allows for a real physical independence between arm transport and hand orientation (hand orientation remains constant when the wrist is displaced along a straight line). This may have induced a decoupling between hand transport and orientation (Desmurget et al. 1996).

\section{Donders' Law}

One of the ways that one postural configuration may be chosen over another is Donders' Law, which, when applied to the arm, suggests that the amount of torsion is a unique function of arm position relative to the body (Crawford and Vilis 1995; Hore et al. 1992; Miller et al. 1992). In most studies of Donders' Law of the arm, torsion is defined as rotation about a body fixed axis that would align with the arm while it points straight ahead. During straight-arm pointing, the upper arm obeys a rule very similar to Listing's Law, which states that torsion is maintained at a constant "zero" value for all pointing directions. This has led some to suggest that the arm-control system might possess a Donders' operator that takes in desired pointing direction and outputs a command for desired threedimensional (3-D) arm orientation (Crawford and Vilis 1995). In contrast to the observations suggesting a consistent and reproducible reduction of the number of degrees of freedom (Crawford and Vilis 1995; Hore et al. 1992; Miller et al. 1992), other authors have reported violations of Donders' Law for the arm (Gielen et al. 1997; Soechting et al. 1995), particularly when the forearm is allowed to move (Medendorp et al. 2000).

\section{Interactions of parallel processing theories and Donders' Law}

Typically, investigations of the role of Donders' Law in arm movements and theories of a higher-order control system integrating the posture of the whole arm have been considered separately. However, both theories ultimately deal with the same kinematics, suggesting that they need to be synthesized at some level. In particular, whereas Donders' Law studies of arm control have focused on pointing movements with the arm straight or bent, reaching and grasping movements must also orient the grasp about a torsional axis aligned with the arm. The fingers themselves could contribute in part to the shaping of grasp orientation but at some level one expects torsional rotations of the arm to come into play. One possible hypothesis is that a "grasp system" governs the lower arm, causing it to rotate torsionally into alignment with the grasp target, while the upper arm is governed by a "reach system" that obeys Donders' Law. There are clues, however, that this may not be the case.

Some authors have argued that the posture of the upper arm is dependent on the orientation as well as the location of a rod which is to be grasped (Desmurget et al. 1996, 1998; Hepp et al. 1992; Soechting and Flanders 1993). For example, Desmurget et al. (1998) found that the posture of the arm was altered at both the distal and proximal levels when the orientation of a target object was changed. In addition, nonhuman primates grasping a cylindrical object whose tilt is varied, tend to orient their proximal arm (shoulder and elbow) so as to restrict the amount of rotation of the forearm required to align the hand with the cylinder (Tillery et al. 1995). These findings imply that the neural transformation from target orientation to hand orientation is not independent of the transformation dealing with target location. The position and orientation of the hand in space are unlikely to be controlled through separate independent neural pathways (Desmurget et al. 1998). A functional coupling may exist between the different components of prehension movements that sustain arm transport and hand orientation by a single, integrated command. Such a command may come from a higher-order control system, which specifies the reaching and grasping movement as a whole and utilizes context-dependent kinematic rules like Donders' Law.

The current investigation focused on whether upper arm (humerus) torsion contributes to grasp orientation in a "typical" reaching and grasping movement. Based on previous studies, one might predict that Donders' Law of the upper arm could hold for smaller ranges of grasp orientations, but that larger target orientation ranges could be subserved by systematic torsional rotations in the upper arm, which would violate strict interpretations of Donders' Law. If so, Donders' Law of the upper arm might break down completely, or it might be modified by the (as yet unknown) kinematic rules that govern arm contribution to grasp. Is upper arm torsion recruited at some quantitative threshold of grasp orientation? Once recruited, does it follow some graded linear or nonlinear rule of upper arm/lower arm (forearm) coordination? Finally, it is not known how such rules would interact with the other aspects of Donders' Law, like the position-dependencies in arm torsion 
that are thought to optimize various aspects of limb control and task demands (Hore et al. 1992; Medendorp et al. 2000; Straumann et al. 1991).

The purpose of this study was to address these questions, thereby re-examining higher-order visuomotor control theories of arm control in the context of theories based on kinematic rules like Donders' Law. To this end, we investigated the contributions of upper and lower arm torsion to grasp orientation during a reaching and grasping movement. We utilized rotation vectors to describe the orientations of the different segments, which represent any position as the result of a virtual rotation from a fixed reference position to the current position. In this description, torsion refers to a rotation about a bodyfixed axis that would align with the arm when it points straight ahead, which can be regarded as "spatial torsion." In experiment 1, subjects reached out and grasped a target block that was presented in 1 of 19 orientations at one position in a vertical presentation board. In experiment 2, target blocks were presented in one of three orientations at nine different positions in the presentation board. If the reach and grasp systems govern the upper and lower arm separately, one would expect the lower arm to contribute exclusively to grasp orientation and the upper arm to obey Donders' Law for all grasp orientations. However, if the latter is not the case, then we need to make a different distinction between reach and grasp rules within the kinematics of arm-hand coordination.

\section{METHODS}

\section{Experiment 1}

SUBJECTS. Six subjects (3 males, 3 females; age range, 27-47 yr old; mean age, $33.3 \mathrm{yr}$ old) with normal or corrected-to-normal vision voluntarily participated in the experiment. Subjects were strongly right-handed, as determined by a modified version of the Edinburgh Handedness Inventory (Oldfield 1971).

EXPERIMENTAL DESIGN. The contribution of upper and lower arm torsion to grasp orientation was investigated by having subjects reach to a rectangular target block $(2.5 \times 10 \mathrm{~cm})$ that was placed in the center $(117 \mathrm{~cm}$ from the floor) of a vertical matte black presentation board $(64 \times 100 \mathrm{~cm})$ mounted on a table $(48 \mathrm{~cm}$ high). Subjects were seated with their torso rotated $45^{\circ}$ leftward with respect to a presentation board ( $55 \mathrm{~cm}$ away from the shoulder) so that the target block was positioned near the center of the arm's mechanical range. To minimize body movement, subjects were strapped into their chair with a seatbelt worn around their chest. The subjects sat with the tips of their index finger and thumb of their right hand on a start button $(80$ $\mathrm{cm}$ high, $25 \mathrm{~cm}$ from the presentation board). Subjects were instructed to reach out quickly, accurately and as "naturally" as possible and grasp the target block across its narrow width. Between trials, subjects kept their eyes closed, while the target block was randomly rotated by a stepper-motor into a new orientation. The orientations ranged from $0^{\circ}$ (horizontal) to $90^{\circ}$ (vertical), in $5^{\circ}$ increments. The testing block of trials consisted of 88 trials: the $0^{\circ}, 45^{\circ}$, and $90^{\circ}$ orientations were each presented eight times; each of the other orientations was presented four times. The subjects initially performed five practice trials. At the end of the experiment, a "calibration" trial was collected with the subject grasping the target block in the horizontal orientation. This calibration trial was used to determine a reference orientation to which all other orientations adopted during the experiment were computed.

DATA COLLECTION. Three 8-mm-diameter infrared light-emitting diodes (IREDs) were attached to an aluminum extension $2 \mathrm{~cm}$ above the distal portion of a subject's right index fingertip; three more IREDs were attached to an aluminum extension mounted between a subject's right thumbnail and the first knuckle. The aluminum extensions were used to allow the infrared-sensitive camera system (OPTOTRAK) an optimal view of IREDs. Ten IREDs were mounted on two wooden crosses - one IRED at the end of each cross arm and one IRED in the middle. One of the crosses was attached to the lower right arm, close to the radius of the wrist, with a Velcro band and medical tape. The other cross was mounted in the same way on the lateral surface of the upper right arm (see Fig. 1A).

The IREDs were monitored by an OPTOTRAK positioned approximately $2 \mathrm{~m}$ from the subject. The 3 -D coordinates of the IREDs were recorded at a rate of $150 \mathrm{~Hz}(100 \mathrm{~Hz}$ for experiment 2$)$, stored by the Optotrak's data acquisition unit, and later filtered off-line (with a low-pass second-order Butterworth filter with a 7-Hz cutoff).

DEPENDENT MEASURES. Custom software was used to compute rotation vectors that represented any instantaneous arm position as the result of a virtual from a fixed reference position to the current position. In the space-fixed right-handed coordinate system, the rotation vector is defined by

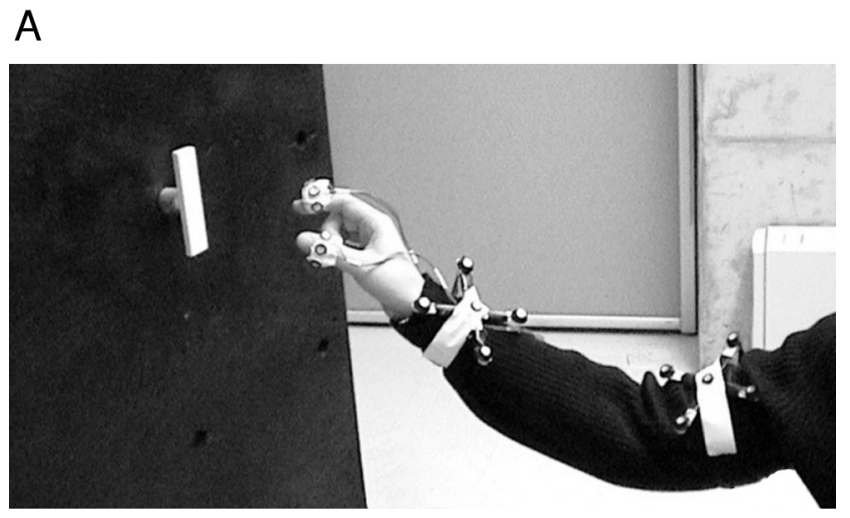

\section{B}

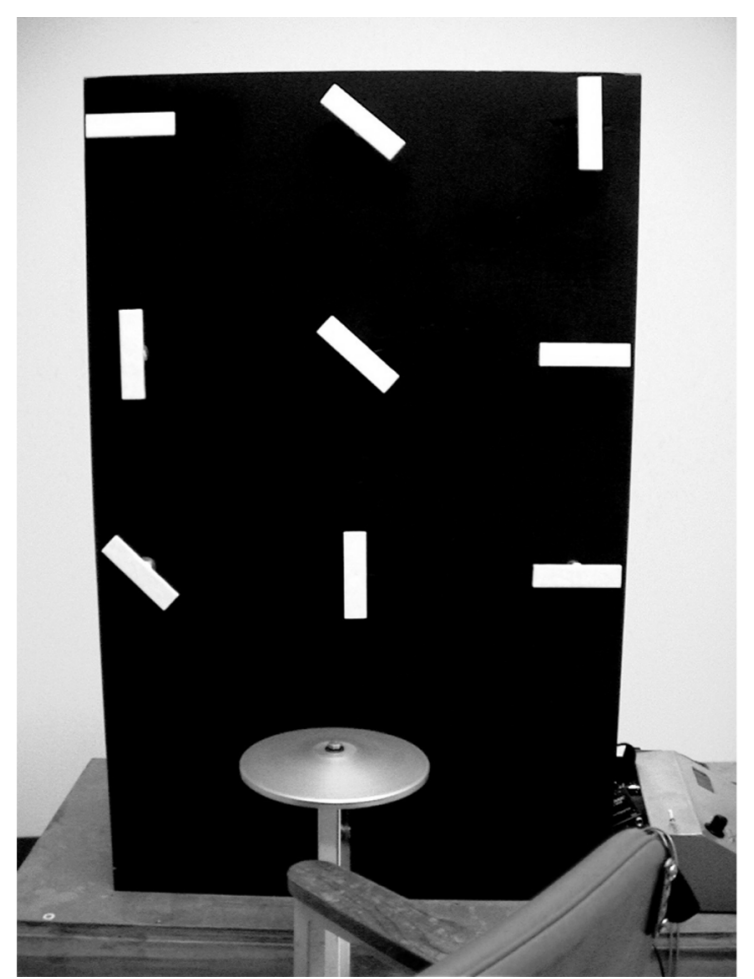

FIG. 1. Photographs of (A) infrared light-emitting diodes (IREDs) position on the hand and arm and the target location in the vertical presentation board used in experiment 1 and (B) the nine target locations used in experiment 2. 


$$
\vec{r}=\tan (\theta / 2) \cdot \vec{n}
$$

where $\vec{n}$ represents the direction of the rotation axis and $\tan (\theta / 2)$ denotes the amount of rotation by an angle $\theta$ about that axis (Haustein 1989). The $x$ component of the rotation vector describes the torsional orientation (clockwise/counter-clockwise) of the arm. The $y$ and $z$ components specify the vertical (up/down) and horizontal (right/left) orientation, respectively. For example, a rotation vector pointing in the positive $x$ direction represents a position obtained by rotating the arm clockwise from the reference position. The rotation vector describing the orientation of the lower arm with respect to the upper arm, $\vec{r}_{\text {lu }}$, was computed using

$$
\vec{r}_{\mathrm{lu}}=\frac{\vec{r}_{\mathrm{ls}}+\vec{r}_{\mathrm{us}}+\vec{r}_{\mathrm{ls}} \times \vec{r}_{\mathrm{us}}}{1-\vec{r}_{\mathrm{ls}} \cdot \vec{r}_{\mathrm{us}}}
$$

where $\vec{r}_{\text {Is }}$ is the first rotation, $\vec{r}_{\text {us }}$ is the second rotation, and $\times$ indicates the vector cross-product.

\section{Experiment 2}

SUBJECTS. Eight subjects voluntarily participated in the experiment (5 males, 3 females; age range, 21-47 yr old; mean age, $29.6 \mathrm{yr}$ old). All subjects were strongly right-handed and had normal or correctedto-normal vision.

EXPERIMENTAL DESIGN. In experiment 2, the torsional contribution of the upper and lower arms and their interaction with Donders' Law was examined across a wider range of grasping space by requiring subjects to reach out and grasp target blocks that were presented in one of three orientations (horizontal, three-quarter, and vertical) at nine different positions in the vertical presentation board (see Fig. $1 B)$. Each presentation position was spaced $25 \mathrm{~cm}$ from its neighbor $\left(50^{\circ}\right.$ presentation angle), in a $50 \times 50 \mathrm{~cm}$ grid. In contrast to experiment 1 , subjects only wore two IREDs on their index finger and thumb. There were six testing block of trials consisting of 18 trials each. Each orientation $\times$ position combination was presented four times (108 trials total). The subjects initially performed five practice trials. At the end of the experiment, a calibration trial was collected with the subject grasping the target block placed at the center position of the presentation board in the $0^{\circ}$ orientation.

DEPENDENT MEASURES. The 3-D patterns of upper and lower arm orientations at the end of the reaching and grasping movement (when arm transport velocity fell below $5 \mathrm{~cm} / \mathrm{s}$ ) were computed for each subject. This was done by fitting a second-order surface to the rotation vector data (Hore et al. 1992; Medendorp et al. 2000; Miller et al. 1992; Straumann et al. 1991; Theeuwen et al. 1993) as follows

$$
r_{\mathrm{x}}=a+b r_{\mathrm{y}}+c r_{\mathrm{z}}+d r_{\mathrm{y}}^{2}+e r_{\mathrm{y}} r_{\mathrm{z}}+f r_{\mathrm{z}}^{2}
$$

in which $r_{\mathrm{x}}, r_{\mathrm{y}}$, and $r_{\mathrm{z}}$ represent the torsional, vertical, and horizontal components of the rotation vectors relative to the reference position. These surfaces were then averaged across subjects to achieve one averaged plane per target orientation. Parameters $b$ and $c$ characterize the orientation of the plane. Parameter $e$ (denoted as the twist score) allows the surface to twist, whereas parameters $d$ and $f$ yield a parabolic curvature in the $r_{\mathrm{y}}$ and $r_{\mathrm{z}}$ direction, respectively. The scatter of the data relative to the fitted surface (commonly denoted as the thickness of the surface) is defined by the SD of the distances of all samples in the $r_{\mathrm{x}}$ direction to the fitted surface. The smaller the thickness, the closer the rotation vectors stay to their surface, and therefore the better Donders' Law is obeyed. These parameters have been previously discussed in detail (Medendorp et al. 2000).

\section{RES U L T S}

\section{Experiment 1}

To investigate the contribution of upper and lower arm torsion to grasp orientation, six subjects were required to reach out and grasp a target block that was oriented to one of 19 orientations at a single location in a vertical presentation board. For each of the six subjects, mean values of all the dependent measures were calculated for each orientation and entered into separate repeated measures ANOVA. Degrees of freedom were corrected according to the Huynh-Feldt adjustment. All tests of significance were based on an $\alpha$ of 0.05 . Post hoc NeumanKeuls analyses were performed where necessary.

LIMB TRAJECTORY-ORIENTATION AS A FUNCTION OF TRANSPORT. Several studies have shown that if Donders' Laws of the eyes, head and arm hold, they only hold for static postures at the end of the movement (Crawford et al. 1999; Glenn and Vilis 1992). Therefore final limb orientation was the focus of our analysis. Before settling into this matter, however, we examined the six-dimensional (3-D rotational and 3-D translational) trajectories of the lower and upper arm to obtain a qualitative picture of how these final orientations arose. Furthermore, this allows us to touch briefly on issues of relative timing. For example, if the upper arm does contribute to grasp orientation, does it do so from the beginning of the reaching movement or after the lower arm meets some mechanical limit? In other words, do limb transport and limb torsion co-evolve during a reaching and grasping movement?

To address this question, one needs a representation of limb trajectories that shows location and orientation separately but simultaneously for both the upper and lower arm. The plane containing the upper and lower arm, sometimes shown in motor control studies, shows upper arm torsion when the arm is bent, but unfortunately is ambiguous for lower arm torsion and for both upper and lower limb torsion when the arm is fully extended. Instead, we have plotted the 3-D position of the IREDs mounted on the wooden crosses attached to the lower and upper arm of a single subject during a reach to a horizontal $\left(0^{\circ}\right)$ target block (see Fig. 2, $A$ and $B$, respectively) and a vertical $\left(90^{\circ}\right)$ target block (see Fig. 2, $C$ and $D$, respectively). A rigid body was formed that "connected" the five IREDs on each wooden cross (see Fig. 1) to form the two planes comprising an "X." In other words, the Xs in Fig. 2 represent the wooden crosses attached to the upper and lower arm on which the IREDs were mounted. Each X was mathematically rotated (Fig. 2) into the plane perpendicular to the long axis of the upper or lower arm, so that the vertex of the $\mathrm{X}$ shows location of the segment and the relative rotation of the $\mathrm{X}$ shows orientation. The position of this $\mathrm{X}$ in space was plotted for each time point during a reach, which allowed for the trajectory and rotation of the lower and upper arm to be tracked through space.

During the $0^{\circ}$ reach, the lower arm underwent a counterclockwise $(\mathrm{CCW})$ torsional rotation immediately after an initial righting stage, which appears to be a default $\mathrm{CW}$ twist that tends to right the palm of the hand with the vertical in response to lifting the arm from its initial resting position, aligning the grasp orientation toward the target's horizontal orientation (see Fig. 2A). The arm maintained this orientation as it approached the target. Note that in this trajectory, the final + position is upright and aligned in the frontal plane because we have defined it thus. In contrast, for the $90^{\circ}$ reach, the lower arm (Fig. 2C) underwent a steady clockwise (CW) torsional rotation throughout the reach, eventually aligning the arm-fixed + close to the frontal plane, but now twisted about $45^{\circ}$ clockwise 
Target $0^{\circ}$

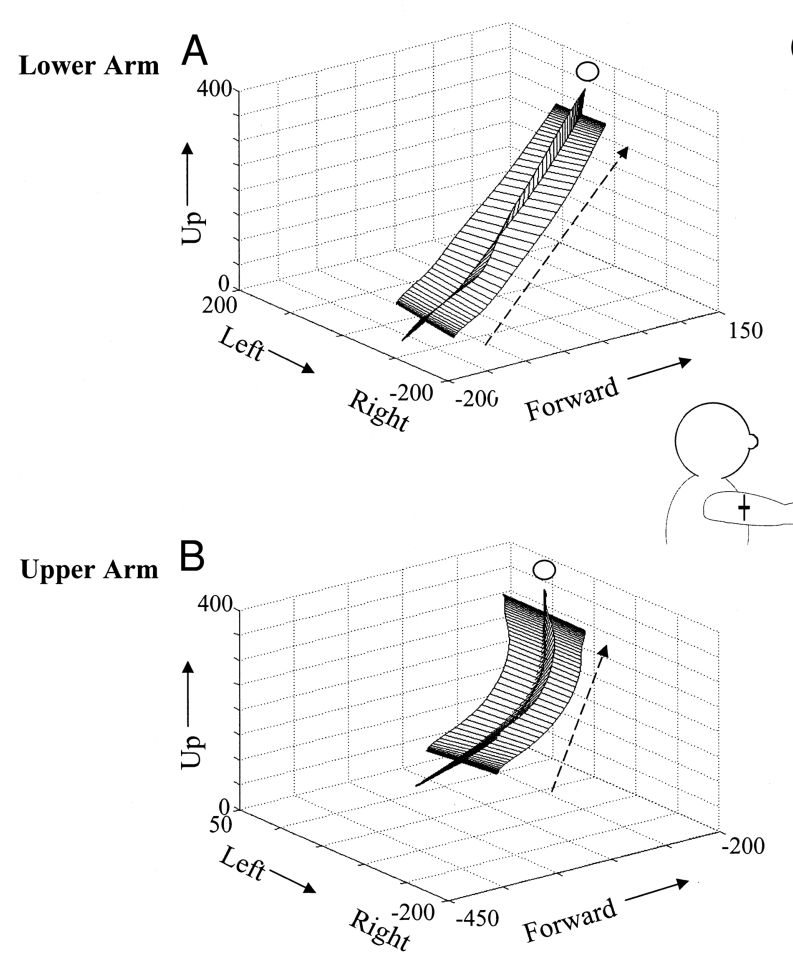

B
Target 90

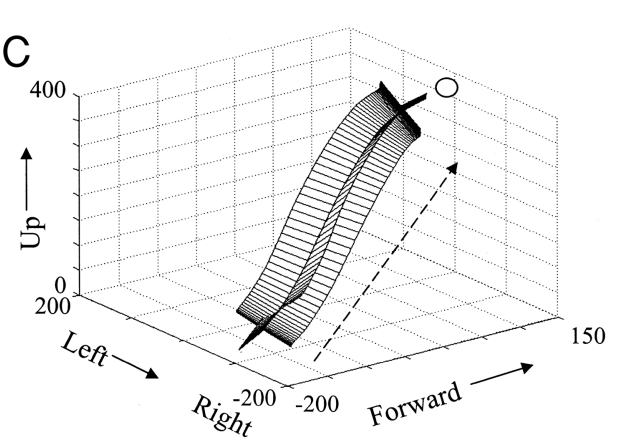

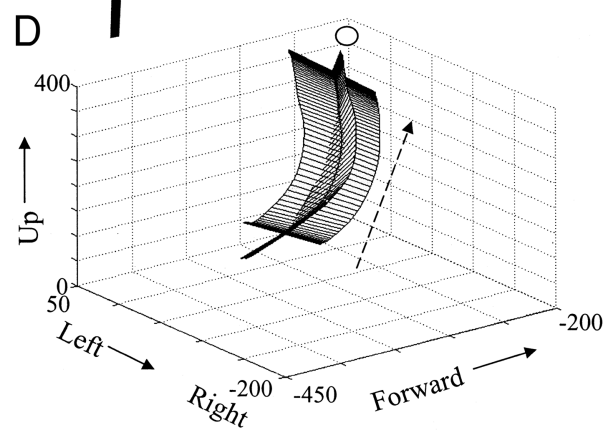

FIG. 2. Three-dimensional trajectories of the IREDs mounted on the wooden crosses attached to the lower and upper arm of a single subject during a reach to a horizontal $\left(0^{\circ}\right)$ target block (A and B, respectively) and a vertical $\left(90^{\circ}\right)$ target $(\mathrm{C}$ and $\mathrm{D}$, respectively) as viewed from behind, right, and above the subject. The endpoints of the $0^{\circ}$ reach for the upper and lower arm were rotated so that the $\mathrm{X}$ was aligned as a cross $(+)$ in the frontal plane when the arm was fully extended toward the $0^{\circ}$ target block, as illustrated by the schematic drawing in the middle. To keep this + fixed within the upper or lower arm, arm orientation information gathered from the entire trajectory was used to keep the $\mathrm{X}$ to + rotation fixed in limb coordinates. This rotation was applied to every point in the trajectory for both the $0^{\circ}$ and $90^{\circ}$ reaches. The vertex of the + shows the location of the segments and the relative rotation of the + shows orientation. Broken line arrows indicate progression through time. One arm on each cross is tagged with a circle to highlight torsional rotation.

relative to the other final position. Clearly, lower limb torsion was planned from an early stage to match, or at least contribute to, the final grasp orientation.

The grasp-dependent torsional rotation in the upper arm was less obvious, as the 6-D trajectories portrayed in Fig. 2, $B$ and $D$, were nearly identical. Nevertheless, it does appear that the upper arm went through a small clockwise torsional rotation as the target block orientation increased from $0^{\circ}$ (see Fig. $2 B$ ) to $90^{\circ}$ (see Fig. 2D). This clockwise rotation developed slowly throughout the reach trajectory, which suggests that the upper arm contributes to grasp orientation from the beginning of the movement. These results suggest that limb transport and limb torsion co-evolve during a natural reaching and grasping movement.

FINAL LIMB TORSION. While the previous trajectory analysis provided information about the movement of the whole arm during the entire course of the reach, the next analysis more carefully examined the contribution of upper and lower arm torsion to final grasp orientation by focusing on movement endpoints. Figure 3 represents the torsional component of the rotation vectors at the end of grasping movements (defined by the transport velocity of the arm dropping below $5 \mathrm{~cm} / \mathrm{s}$ ) for the upper and lower arm and the lower arm with respect to the upper arm. Recall that torsion was measured relative to the amount of torsion taken at a reference position with the arm extended and the hand grasping the target block at the horizontal $\left(0^{\circ}\right)$ orientation.

As the required grasp orientation increased from $0^{\circ}$ to $90^{\circ}$ (Fig. 3), there was a significant clockwise torsional rotation in the upper arm $\left(\square\right.$; range of $8.0^{\circ}$ in space, $F_{(18,90)}=26.25, P<$

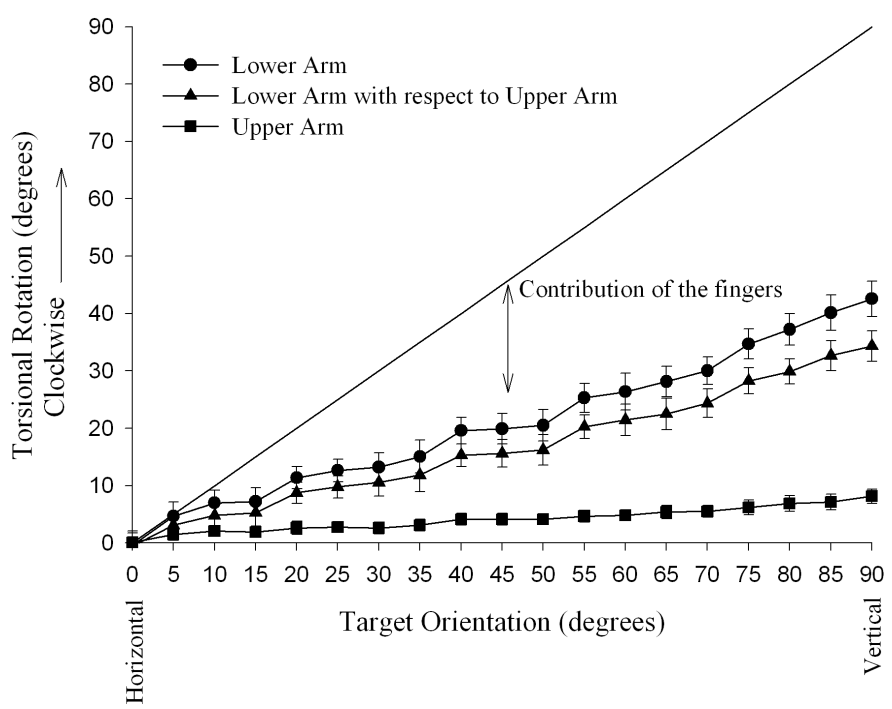

FIG. 3. Effects of target orientation on the torsional rotation of the arm (e, lower arm; $\mathbf{\Lambda}$, lower arm with respect to upper arm; $\mathbf{n}$, upper arm). 


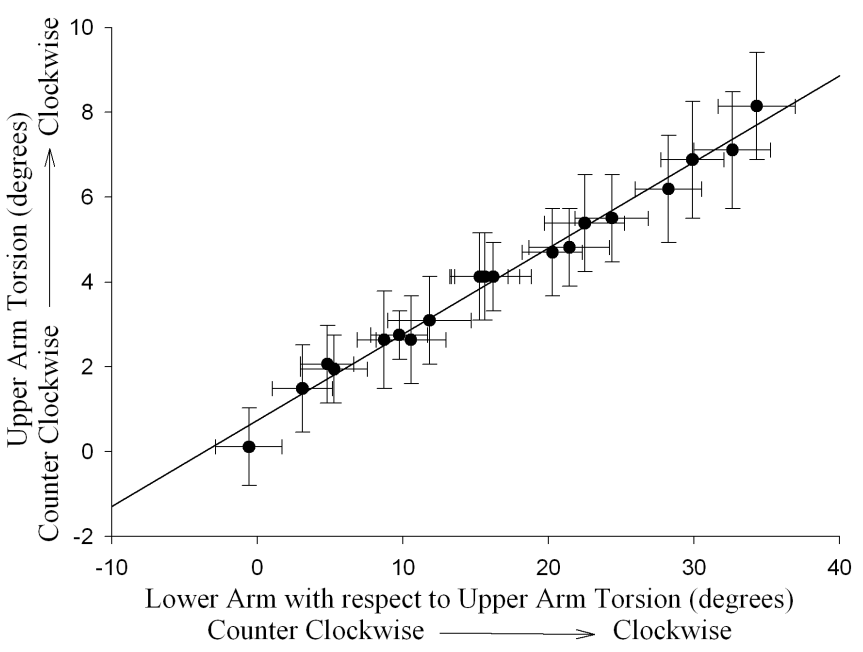

FIG. 4. Upper arm torsion plotted as a function of lower arm with respect to upper arm torsion. Error bars $=\mathrm{SE}$.

$0.001)$ and lower arm with respect to the upper arm $(\mathbf{\Delta}$; range of $34.9^{\circ}$ with respect to upper arm, $F_{(18,90)}=133.99, P<$ $0.001)$, and the sum of these two, lower arm in space $(\mathbf{O}$; range of $43.0^{\circ}$ in space, $\left.F_{(18,90)}=166.86, P<0.001\right) .{ }^{1}$ The line with a slope of 1 represents the total torsional rotation needed to appropriately orient the grasp for the rotated target block. Clearly, both the upper arm and lower arm contributed to grasp orientation, although to different extents.

It is also clear that the torsional rotations generated by the upper and lower arm did not account for all the torsion needed to achieve the final grasp orientation. Perhaps surprisingly, arm torsion only accounted for about one-half of the re-orientation required to align grasp with the target block. By process of elimination, the formation of the hand and fingers in the grasp must, therefore, make up a large portion of the required torsional rotation. Based on these results, it appears that the torsional rotations of the upper and lower arms and the fingers forming the grasp are combined to achieve the final required orientation of the grasp.

LIMB COORDINATION. Does the upper arm only contribute to final grasp orientation when some target orientation threshold is met or does a constant ratio exist between upper and lower arm torsion? Figure 4 plots average upper arm torsion as a function of average lower arm torsion (across trials and then across subjects) for our 19 target orientations. This figure shows that a very simple linear relationship exists across subjects between the upper arm torsion and the torsional rotation of the lower arm with respect to the upper arm, with an intercept close to $0(y=0.209 x+0.7412 ; r=0.99, P<$ 0.001). As the torsion of the lower arm increases, so does the torsion of the upper arm. This suggests that the upper and lower arm rotate in coordination with one another to achieve the torsion necessary to successfully orient the grasp for any target orientation.

It should be noted that the horizontal and vertical components of the rotation vectors were constant and close to zero, for both the upper arm and lower arm, when subjects reached to the central target. So here, the variations in final orientation

\footnotetext{
${ }^{1}$ The intersubject variability was marginal on all of our measures, as indiated by the SE in the within-subjects designs.
}

were one-dimensional (about the torsional axis). The same relationship would have been observed, therefore, irrespective of the coordinates used (e.g., Fick angles, Euler angles, or rotation vectors).

\section{Experiment 2}

In experiment 2, the 3-D rotations of the upper and lower arm and their interaction with Donders' Law were examined across a wider range of grasping space by requiring subjects to reach out and grasp target blocks that were presented in one of three orientations (horizontal, three-quarter, and vertical) at nine different locations in grasping space. This allowed us to quantify Donders' Laws of the arm across a range of target locations, examine the contribution of arm torsion to grasp across these locations, and look for interactions between these two factors.

For each of the eight subjects, mean values of the rotation vectors' endpoints for the upper arm, the lower arm, and the lower arm with respect to the upper arm were calculated for every combination of target orientation and target location. The mean values were entered into separate $3 \times 3 \times 3$ (target orientation $\times$ target row $\times$ target column) repeated measures ANOVA. Degrees of freedom were corrected according to the Huynh-Feldt adjustment. All tests of significance were based on an $\alpha$ of 0.05 . Post hoc Neuman-Keuls analyses were performed where necessary.

EFFECTS OF TARGET POSITION AND ORIENTATION ON 3-D ARM ROTATIONS. What are the combined effects of target location and orientation on the 3-D rotations of the lower and upper arm? Figure 5 represents the average (across trials and then across subjects) vertical, horizontal, and torsional components of lower arm orientation in space (Fig. 5, $A-C$ ) and upper arm orientation in space (Fig. 5, $D-F$ ), at the end of grasping movements for each target location and orientation. Data are plotted from three different "views" (Fig. 5, $A$ and $D$, vertical vs. horizontal; $B$ and $E$, torsion as a function of vertical; $C$ and $F$, torsion as a function of horizontal) and grouped by grasp target orientation, i.e., solid ellipses are fit to data for the $0^{\circ}$ targets (black symbols), dotted ellipses are fit to the $45^{\circ} \mathrm{CW}$ targets (white symbols), and dashed ellipses are fit to the $90^{\circ}$ targets (gray symbols). Symbols are keyed according to the vertical position (circles for downward targets, squares for upward targets, and triangles for intermediate targets).

From the left panels of Fig. 5, one can see that upper and lower arm orientations make different contributions to acquire target location, and as one might expect, these components only change slightly with grasp orientation. As the target orientation changes from horizontal to vertical, there is a slight leftward trend for all target locations. Perhaps because the $\mathrm{CW}$ torsion required to successfully grasp the targets at the $45^{\circ}$ and $90^{\circ}$ orientations rotates the elbow to the left when the arm is not totally extended and perhaps also to compensate for changes in finger/hand location that we did not measure.

More importantly, the horizontal and vertical plots against torsion reveal a dependence of torsion on horizontal and vertical arm position that is also affected by grasp orientation. This is most clearly seen for the lower arm in Fig. $5 C$ and for the upper arm in Fig. $5 E$. From these perspectives, both the average lower arm and upper arm orientation range forms a torsionally compact, but tilted distribution for a given grasp 
A

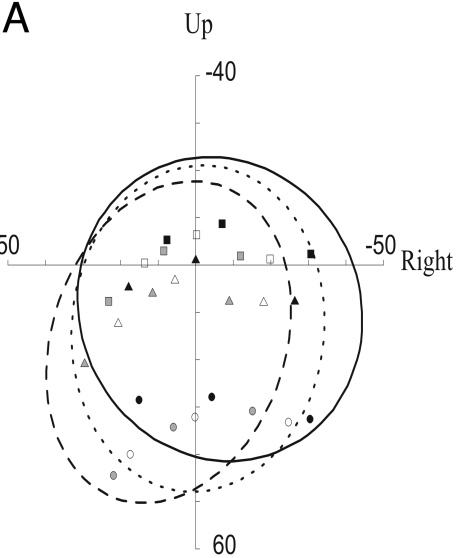

D

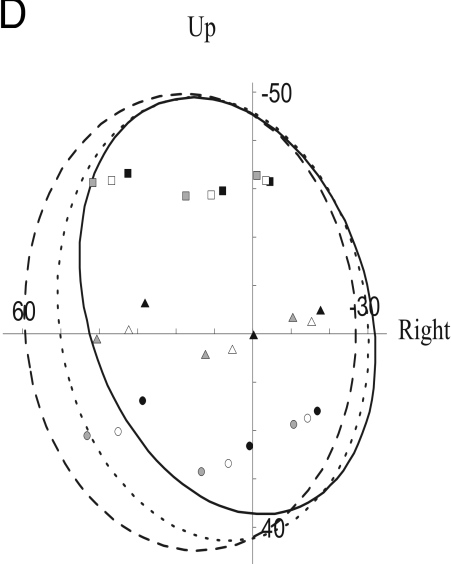

B

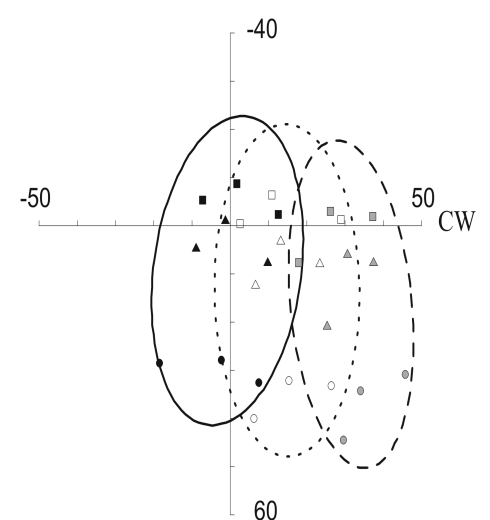

$E$

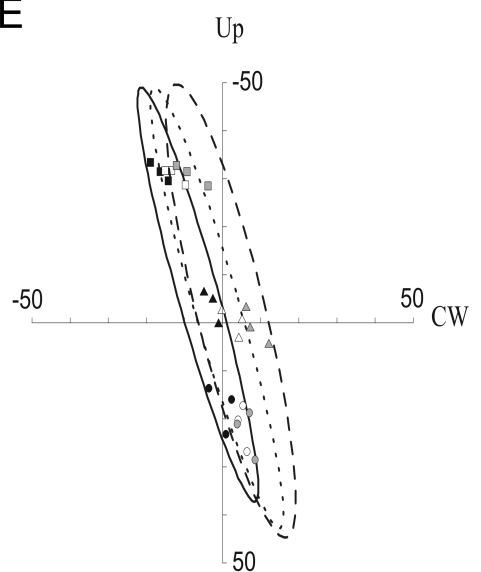

C

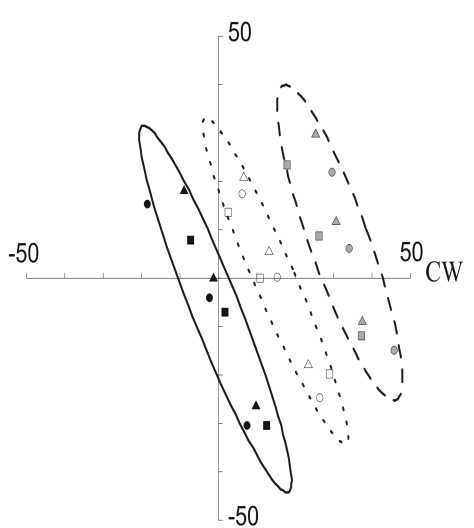

$\mathrm{F}$

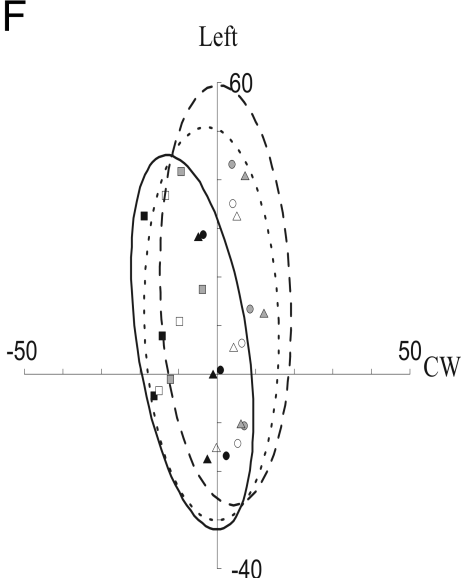

FIG. 5. Effects of target position and orientation on vertical, horizontal, and torsional components of the rotation vectors of the arm at the end of a grasping movement. $A$ : vertical rotation as a function of horizontal rotation for the lower arm. $B$ : vertical rotation as a function of torsion rotation for the lower arm. $C$ : horizontal rotation as a function of torsional rotation for the lower arm. $D$ : vertical rotation as a function of horizontal rotation for the upper arm. E: vertical rotation as a function of torsion rotation for the upper arm. $F$ : horizontal rotation as a function of torsional rotation for the upper arm $[\square$, upper target positions; $\mathbf{\nabla}$, middle target positions; $\bigcirc$, lower target positions; black symbols, horizontal $\left(0^{\circ}\right)$ target block orientation; white symbols, three-quarter $\left(45^{\circ}\right)$ target orientation; gray symbols, vertical $\left(90^{\circ}\right)$ target orientation].

orientation (i.e., the ellipses in Fig. 5, $C$ and $E$, are torsionally narrow but tilted). Torsion varies systematically with horizontal position in the lower arm-in-space (Fig. 5C) and as a function of vertical position for the upper arm (Fig. 5E). As a result of these tilts, these ranges appear to be less compact when viewed from the perspectives in Fig. 5, $B$ and $E$. However, these ranges shift torsionally with grasp orientation. As in experiment 1 for one grasp location, the entire grasp range shifted progressively $\mathrm{CW}$ with the $45^{\circ}$ and $90^{\circ}$ targets, but not by as much as the targets, and to a much greater extent for the lower arm than the upper arm. In the remaining sections we will quantify the amount of this shift, the affect of this shift on the shape of the orientation range (i.e., the dependence of torsion on vertical and horizontal components) and the torsional scatter between individual trials.

LIMB TORSION. How does upper and lower arm torsion contribute to final grasp orientation across different grasp orientations? In Fig. 6, we have averaged the torsional data across target position to highlight the significant clockwise torsional rotation in the upper arm $\left(\square\right.$; range of $8.5^{\circ}$ in space, $F_{(2,14)}=$ 84.46, $P<0.001)$ and the lower arm $\left(0\right.$; range of $32.3^{\circ}$ in space, $F_{(2,14)}=225.74, P<0.001 ; \boldsymbol{\Delta} ; 25.8^{\circ}$ with respect to upper arm, $\left.F_{(2,14)}=133.47, P<0.001\right)$. These results again suggest that the amount of torsional offset changes systematically for both the upper and lower arm to bring the hand in the

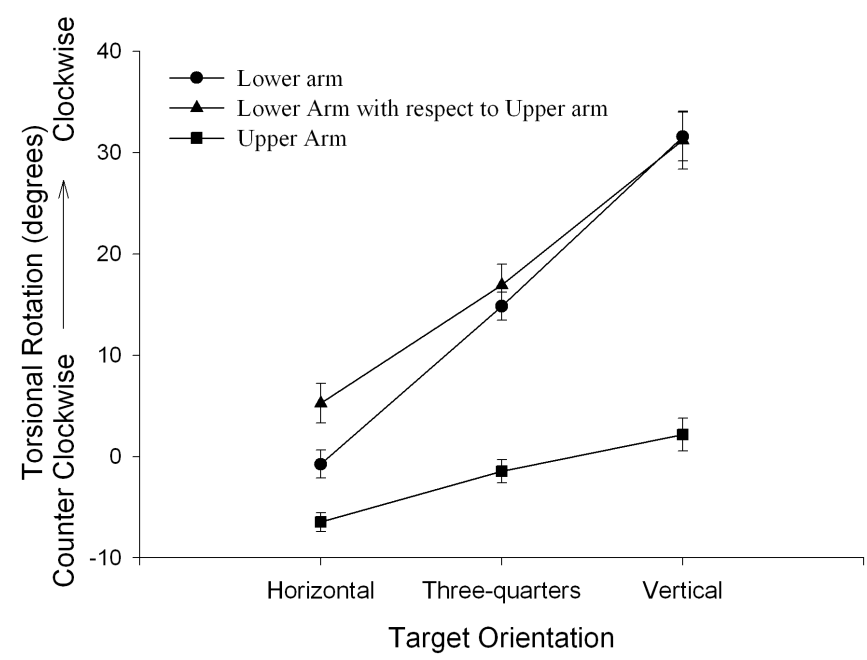

FIG. 6. Effects of target orientation on torsional rotation of the arm (e, lower arm; $\mathbf{\Delta}$, lower arm with respect to upper arm; $\mathbf{n}$, upper arm). 
appropriate final grip orientation. On a global scale (i.e., across all target orientations), Donders' Law is systematically violated for both the upper and lower arm. While Donders' Law is violated across target orientations, do kinematic rules like Donders' Law hold for a subset of the task demands (Medendorp et al. 2000)? If a torsional offset is selected for a particular target orientation, would the location of the target in the workspace affect this selection? In other words, how would target location and orientation interact to affect the selection of arm torsion throughout the grasping work-space?

DONDERS' SURFACES. To test the validity of Donders' Law for both the upper and lower arm for a given grasp orientation, we fitted a second-order surface (see Eq. 3) to the movement endpoint data for each subject generated when grasping targets at various locations. We did this separately for grasps to the $0^{\circ}$, $45^{\circ}$, and $90^{\circ}$ target orientations. This was done for both the upper arm and lower arm in space. It was not possible to fit a surface to the movement endpoints produced by the lower arm with respect to the upper arm, since they do not yield the 2-D horizontal-vertical range required to compute a Donders' surface. The optimal parameters of these fits (averaged across subjects) and the corresponding torsional SD of individual trials from these best-fit surfaces are presented in Table 1 for the upper arm and Table 2 for the lower arm. SE measures provide a measure of consistency between the subjects. By using a repeated-measures ANOVA, we determined whether there were significant differences in the parameter values across target orientation. The significance level, $P$, is given in the bottom row of Tables 1 and 2 .

Depictions of the average surface fits for each orientation are shown in Figs. $7 A$ (for the upper arm) and $8 A$ (for the lower arm). These "average fits" were obtained by averaging the parameters of the surface fit and then plotting torsion as a function of the resulting parameters. These ideal surfaces show the systematic dependence of limb torsion on limb direction, roughly corresponding to target direction. Here, torsion is plotted as a function of horizontal position, corresponding to the scatter plots in Fig. 5, $C$ and $F$. In general, one can observe that, for a given grasp orientation, the average surface is relatively flat (albeit with certain tilts and slight curvatures which we will consider later). The surfaces do not appear to show the characteristic "bow-tie" twist observed in the Donders' surfaces for head rotations (Ceylan et al. 2000; Crawford et al. 1999; Glenn and Vilis 1992). Rather, in general, they look more like "Listing's planes," as observed previously for the arm (Gielen et al. 1997; Medendorp et al. 2000).

Moreover, for both the upper (Fig. 7) and lower (Fig. 8) arm, these surfaces look quite similar for each of the three target orientations, only shifted torsionally. As target block orientation changed from horizontal to vertical, the corresponding arm Donders' surfaces shifted in the positive torsional direction
(CW direction) for both the upper arm (see Fig. 7A) and lower arm (see Fig. 8A). These torsional shifts, represented by fit parameter $a$, are depicted in Fig. $7 B$ for the upper arm and Fig. $8 B$ for the lower arm. This parameter reflects the torsional offset in both upper and lower arm, which as we have seen, was required by the system to adopt the appropriate grasp posture. However, the torsional score $(a)$ was the only fit parameter that differed across target orientation. The other five parameters, characterizing the shape of the surface, remained invariant among the three target orientations. This shape invariance suggests that, once a torsional offset was selected, subjects used a constant torsional control strategy when grasping targets at different locations in grasping space.

Coefficients $b$ and $c$ characterize the orientation of the surface. These parameters specify the linear relationships that exist between torsion and vertical arm orientations $(b)$ and torsion and horizontal arm orientations $(c)$. For the upper arm, coefficient $b$ was virtually constant, ranging from 0.26 to 0.31 , and reflects its tendency to roll clockwise when grasping downward targets and $\mathrm{CCW}$ when grasping upward targets. While not significant, the $c$ scores for the upper arm do show some variance across target conditions. For the lower arm, the $b$ scores showed more variability, although not significant, than they did in the upper arm, while the $c$ scores ranged between -0.45 and -0.63 . For the lower arm, this indicates that the arm rotated in a $\mathrm{CW}$ torsional direction when pointing to the right and in a CCW direction when pointing to the left, seen from the position in which the subject grasped at the horizontal target.

Parameters $d, e$, and $f$ describe the curvature of the surface. For the lower arm, these values, while not significant, were quite variable across target conditions, indicating a nonsystematic effect of target orientation on the curvature in the fitted surfaces. For the upper arm, however, the curvature parameters had consistent negative values for all three tested target orientations. Negative values of the $d$ and $f$ scores imply CCW torsion of the upper arm, when pointing in either direction in grasping space. The $e$ parameter, which was also always negative for the upper arm, specifies the twist of the upper arm's surface. The effect of this interaction term depends on where the arm pointed in space. That is, it has a $\mathrm{CCW}$ effect when the upper arm is pointed in down-left or up-right directions and a $\mathrm{CW}$ contribution when the upper arm is pointed in either the down-right or up-left directions. But again, none of these higher-order parameters showed any statistically significant dependence on target orientation.

Finally, Figs. $7 C$ and $8 C$ show the average torsional deviations (thicknesses) from the fitted surfaces for the upper and lower arm, which quantifies the goodness-of-fits of our surfaces. The thickness values for each target orien-

TABLE 1. Coefficients of the fitted 2-D surfaces to upper arm end points

\begin{tabular}{|c|c|c|c|c|c|c|c|}
\hline Orientation & $\mathrm{a}$ & $\mathrm{b}$ & $\mathrm{c}$ & $\mathrm{d}$ & $\mathrm{e}$ & $\mathrm{f}$ & $\sigma(\operatorname{deg})$ \\
\hline 0 & $-0.01 \pm 0.01$ & $0.26 \pm 0.05$ & $-0.05 \pm 0.04$ & $-0.86 \pm 0.15$ & $-0.37 \pm 0.08$ & $-0.17 \pm 0.17$ & $3.15 \pm 0.42$ \\
\hline 45 & $0.03 \pm 0.01$ & $0.31 \pm 0.05$ & $0.09 \pm 0.11$ & $-0.85 \pm 0.15$ & $-0.27 \pm 0.15$ & $-0.25 \pm 0.24$ & $2.58 \pm 0.42$ \\
\hline 90 & $0.07 \pm 0.01$ & $0.26 \pm 0.06$ & $0.17 \pm 0.07$ & $-1.09 \pm 0.12$ & $-0.32 \pm 0.05$ & $-0.51 \pm 0.16$ & $3.87 \pm 0.71$ \\
\hline$P$ & $<0.001$ & $>0.05$ & $>0.05$ & $>0.05$ & $>0.05$ & $>0.05$ & $>0.05$ \\
\hline
\end{tabular}

Values are mean $\pm \mathrm{SD}$ 
TABLE 2. Coefficients of the fitted 2-D surfaces to lower arm end points

\begin{tabular}{|c|c|c|c|c|c|c|c|}
\hline Orientation & $\mathrm{a}$ & B & $\mathrm{c}$ & $\mathrm{d}$ & $\mathrm{e}$ & $\mathrm{f}$ & $\sigma$, deg \\
\hline 0 & $-0.02 \pm 0.01$ & $0.00 \pm 0.17$ & $-0.45 \pm 0.06$ & $-0.72 \pm 0.50$ & $-0.11 \pm 0.38$ & $0.02 \pm 0.48$ & $5.81 \pm 0.45$ \\
\hline 45 & $0.12 \pm 0.01$ & $0.27 \pm 0.12$ & $-0.63 \pm 0.08$ & $-0.64 \pm 0.27$ & $0.58 \pm 0.13$ & $0.38 \pm 0.26$ & $5.34 \pm 0.73$ \\
\hline 90 & $0.28 \pm 0.20$ & $0.22 \pm 0.07$ & $-0.54 \pm 0.09$ & $0.14 \pm 0.30$ & $-0.26 \pm 0.45$ & $0.47 \pm 0.62$ & $6.26 \pm 0.75$ \\
\hline$P$ & $<0.001$ & $>0.05$ & $>0.05$ & $>0.05$ & $>0.05$ & $>0.05$ & $>0.05$ \\
\hline
\end{tabular}

Values are mean $\pm \mathrm{SD}$

tation did not differ significantly across different target orientations for either the upper or lower arm $(P>0.05)$. This means that not only did the Donders' surfaces have the same shape for a given grasp orientation, the degree of adherence to Donders' Law remained the same in each case. For all target orientations, the average thickness of the fitted planes was close to $3^{\circ}$ for the upper arm, indicating small torsional deviates from the fitted surface. For the lower arm, the thickness was close to $5^{\circ}$, which suggests that the accuracy with which Donders' Law is obeyed is better for the upper than for the lower arm.

\section{I S C U S S I O N}

The purpose of the current study was to investigate the contribution of upper and lower arm torsion to grasp orientation during a reaching and grasping movement. This investigation took the approach of combining theories of Donders' Law with those of a higher-order control system integrating the components involved in grasping. The hypotheses were that if Donders' Law holds for the upper arm and if the lower arm alone contributes torsionally to grasp orientation, these differing kinematics could be used a signatures for separate reach and grasp modules of the control system. If both the upper arm and lower arm contribute to
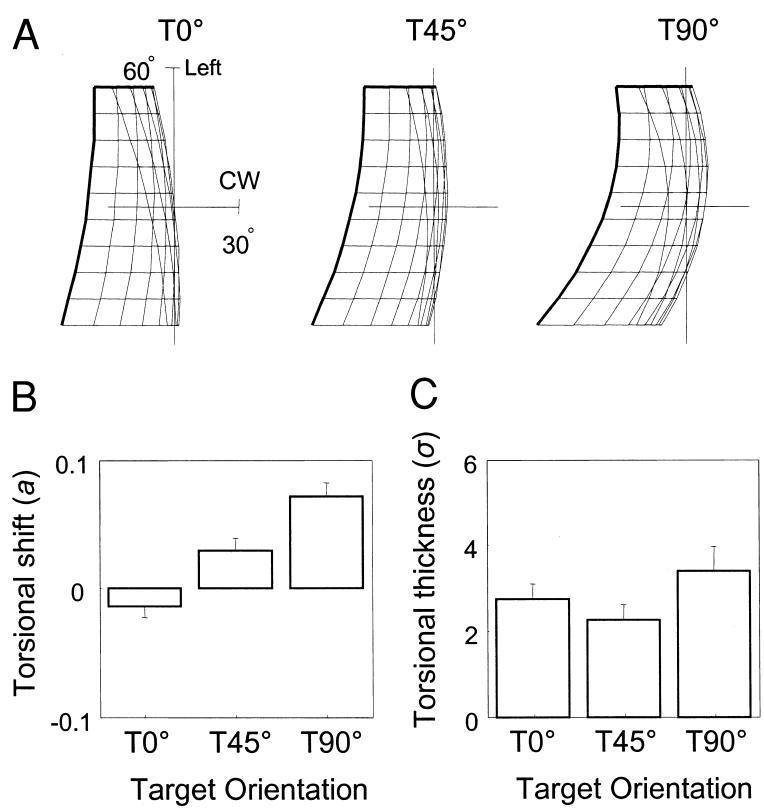

FIG. 7. Mean results from all subjects by characterizing the arm rotation vectors at the end of a movement for the 9 target locations and 3 target orientations. $A$ : mean surfaces generated for each target orientation for the upper arm. $B$ : torsional shift scores. $C$ : torsional thickness for each target orientation for the upper arm. Error bars $=\mathrm{SE}$. grasp orientation, however, this would suggest motor commands based on sensory information about target location and orientation are distributed to the proximal and distal limb through a more complex, coordinated set of kinematic rules. In both experiments, as the required grasp orientation increased from horizontal to vertical, there was a significant clockwise torsional rotation in both the upper and lower arms. Thus it appears that the torsional rotation of the upper and lower arms, and the fingers forming the grasp, are combined to achieve the final required grasp orientation.

While differences in limb segment lengths between subjects might cause some differences in the configurations of joint angles for a given grasp target position, the effect would be minimal and primarily affect the "pointing direction" of the lower and upper arm-not the focus of the current study. Instead, we were interested in the kinematic principals of coordination between the upper arm and forearm torsion related to grasp orientation, which in our representation does not depend on the length of the line segments.

\section{Kinematic rules for grasp orientation}

One approach to understanding limb movement control is to develop a complete model that can predict arm kinematics and dynamics for any arbitrary task situation. Such a model would not only have descriptive and predictive power, it could be

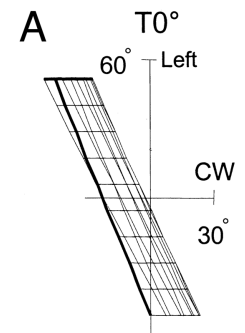

B

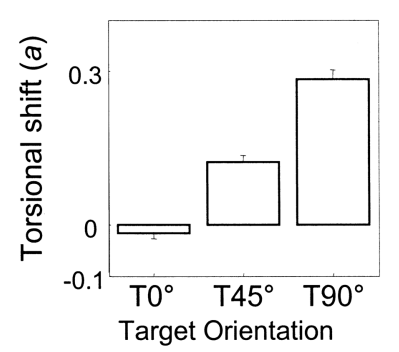

$\mathrm{T} 45^{\circ}$

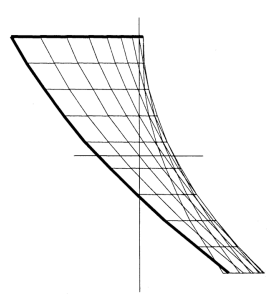

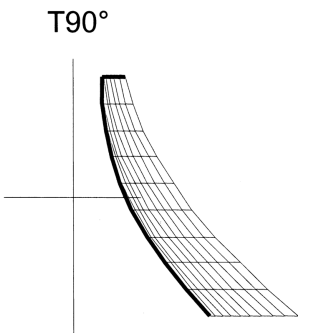

C

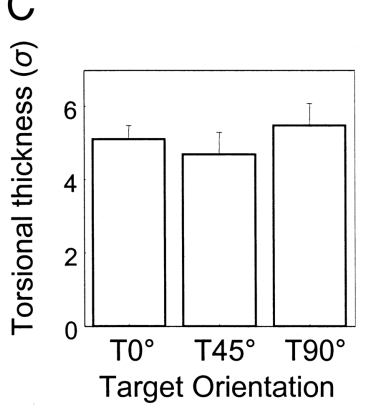

FIG. 8. Mean results from all subjects by characterizing the arm rotation vectors at the end of a movement for the 9 target locations and 3 target orientations. $A$ : mean surfaces generated for each target orientation for the lower arm. $B$ : torsional shift scores, $C$ : torsional thickness for each target orientation for the lower arm. Error bars $=\mathrm{SE}$. 
used to guide invasive physiological investigations into brain function. Toward such ends, motor control investigations have described kinematic rules relating hand path to curvatures in joint space (Desmurget et al. 1998), velocity-position relationships (Ceylan et al. 2000), velocity-accuracy relationships (Fitts 1954), and situations where dynamic considerations override optimal kinematics (Soechting et al. 1995), and vice versa (Flanders et al. 2003). Following this tradition, we add the finding that both the upper arm and lower arm contribute to grasp orientation, that together these two only make up about one-half of the required orientation change (leaving the rest to finger configuration), and to this end, upper arm torsion is linearly coordinated with lower arm torsion relative to the upper arm, providing about one-fifth of the arm's total contribution to grasp orientation.

Our findings that the torsional rotation of the upper and lower arms and the fingers forming the grasp are combined to achieve the final required grasp orientation supports those of Desmurget et al. (1996), who revealed that subjects spontaneously used all the degrees of freedom available in the arm when reaching to objects presented in different orientations. In their task, both the proximal (orientation of the plane of the arm with respect to the vertical) and the distal (prosupination angle) segments were combined to ensure the final orientation of the hand. In contrast to Lacquaniti and Soechting (1982), who argued that forearm rotation does not develop synchronously with the motion of the other two arm joints, our finding of a linear relationship between upper arm torsion and lower arm with respect to upper arm torsion supports the argument that a functional coupling exists between the different components of prehension movements and that a higher-order control system is responsible for their integration. Prehension movements are programmed, from an initial configuration, to smoothly reach a final posture that corresponds to a given location and orientation (Desmurget et al. 1996).

According to this view, the fingers, wrist, elbow, and shoulder are controlled "as a whole" through the computation of a final posture and sustained by a single, integrated command specifying those movements. Our direct finding that lower arm torsion (with respect to upper arm) contributes nearly five times as much as upper arm torsion to grasp orientation, and indirect finding that finger shaping accounts for even more, probably does not reflect a fundamental distal-proximal distinction in the neural control system, but rather a simple work optimization principle: it takes less work to move the fingers than to rotate the wrist, and it takes less work to rotate the wrist than it does to rotate the whole arm about the shoulder. However, the use of all three optimizes the entire working range over which this system can function.

\section{Donders' Law and grasp orientation}

In the current investigation, we also had the opportunity to relate these new rules to a well-known rule that describes some aspects of arm kinematics: Donders' Law. The large torsional shifts that occurred with the change in target orientation from horizontal to vertical suggests that taskspecific violations of Donders' Law occur when grasp formation is essential for the successful completion of a move- ment. However, Donders' Law of the upper arm appears to hold for similar grasp orientations across grasping space, at least as well as it does for pointing movements in human subjects (Gielen et al. 1997; Hore et al. 1992; Medendorp et al. 2000; Miller et al. 1992; Straumann et al. 1991). Moreover, the shape of the Donders' surfaces did not change significantly for different grasp orientations. In other words, the subjects appear to be using a constant location-dependent torsional control strategy when grasping targets at different locations in grasping space.

This finding agrees with the original finding of Hore et al. (1992) that Donders' Law of the arm does not change for different initial orientations in arm torsion during pointing movements. Based on these findings, it appears that whatever biomechanical, gravitation, neural, or task constraints determine the shape and adherence to Donders' Law of the arm during pointing and grasping movements, they are not significantly altered by, nor do they interact with, offsets in limb torsion in the range that these two studies measured.

One way to view these findings, rather than beginning with Donders' Law and then calling the torsional shifts we observed violations of Donders' Law, is to consider that there are more general laws governing arm kinematics that only provide Donders' Law under restricted task circumstances, like a constant set of grasp orientations. This corresponds with the findings reported by other researchers when investigating more "gaze-like" pointing movements (Crawford and Vilis 1995; Medendorp et al. 2000). For example, Medendorp et al. (2000) found that Donders' Law does not hold across all pointing tasks for upper arm movements. In fact, they found that upper arm torsion varied widely for a given pointing target. Torsional variance was considerably reduced, however, for movements with one static elbow configuration, like straight-arm pointing, suggesting that Donders' Law is obeyed for certain task conditions. Medendorp et al. (2000) concluded that while Donders' Law is an important governing principle for the control of arm movements, its various forms may only be limited manifestations of a more general set of task-dependent kinematic rules. In other words, there are kinematically redundant tasks for which Donders' law is obeyed but there are also tasks that constrain more dimensions so that Donders' law will be violated. If this is so, how would these rules be governed by higher-order visuomotor control systems?

\section{Mapping parallel visuomotor modules onto the limb control system}

Consistent with our findings, previous research has suggested that all three conceptual components of a grasping movement (transportation, rotation, and opening of the hand) have access to a common visual representation of the object's orientation (Mamassian 1997). The parietal cortex is thought to transform visual information into the motor commands required for reaching and grasping movements (Connolly et al. 2000; Snyder et al. 2000). In fact, different cortical areas within the parietal lobe have been found to represent plans for different actions. These areas receive inputs from visual extrastriate areas and somatosensory areas that project to dorsal premotor cortex-providing a 
gateway between sensory and motor areas (Caminiti et al. 1996). For example, a medial and posterior area in the parietal lobe [the parietal reach area (PRR)], which includes the medial intraparietal area (MIP) and the occipital parietal area (PO), appears to be specialized for reaching movements (Andersen et al. 1998).

However, detailed electrophysiological and functional MRI (fMRI) recordings in this area suggest a high degree of specialization in parallel modules. A group of PRR neurons collectively represents a specific region in space that is the target of an intended arm movement (Batista et al. 1999). In contrast, neurons in the anterior intraparietal area (AIP) have been shown to be active during grasping (Gallese et al. 1994; Murata et al. 1996, 2000; Taira et al. 1990). These neurons are involved in constructing an action-oriented representation that translates visual information into hand movements - matching the pattern of movement with the spatial characteristics of the object to be manipulated (Sakata et al. 1997).

How would signals from these parallel modules be mapped onto the different kinematics aspects of reaching-grasping examined in the current investigation? Clearly, our work contradicts a distal-proximal distinction that might reflect this modular separation: even the upper arm contributes to grasp orientation. For this and other reasons, it seems more likely that parietal regions MIP and AIP encode higher level "goals," like desired hand location and grasp orientation, respectively, leaving the details of kinematics for downstream motor areas like primary motor cortex. In another sense, however, we did see a kinematic separation that could reflect these two different elements of the early visuomotor system: grasp orientation did not significantly interact with the location-dependent aspects of Donders' Law.

This could mean that a higher level grasp orientation command from parietal cortex (perhaps represented in AIP) is mapped onto a motor control system that implements the rules for coordinated grasp orienting like those we found here, whereas the higher level reach goal commands (perhaps represented MIP) maps more directly onto the positiondependent rules that result in Donders' Law. This is consistent with a recent suggestion that subcortical structures handle the bulk of the position-dependent transformations required for Donders' Laws of the eyes, head, and arm, when their degrees of freedom are underspecified, but can be superseded by cortical orientation commands when this becomes part of the task (Martinez-Trujillo et al. 2003).

In summary, this study has presented evidence that the upper and lower arms and fingers forming the grasp all rotate in coordination with one another to achieve the torsion necessary to successfully orient the grasp. Furthermore, although these kinematic rules of coordination lead to taskspecific violations of Donders' Law, they do not affect the position-dependent aspects of Donders' Law that are observed for a given grasp orientation. Thus the distinction between higher level visuomotor codes for reach location and grasp shape is not so reflected in proximal-distal kinemics as one might expect but may be more closely reflected in the distinction between whole-arm coordination rules for grasp and the position-dependent kinematic rules that result in Donders' Law.

\begin{tabular}{|c|c|c|c|}
\hline Limb & Rotation & Factor & \\
\hline \multirow[t]{9}{*}{ Upper arm } & \multirow[t]{3}{*}{ Torsion } & Orientation & $F_{(2,14)}=84.46, \quad P<0.001$ \\
\hline & & Target row & $F_{(2,14)}=49.19, \quad P<0.001$ \\
\hline & & Target column & $F_{(2,14)}=4.21, \quad P<0.05$ \\
\hline & \multirow{3}{*}{ Vertical } & Orientation & $F_{(2,14)}=43.99, \quad P<0.001$ \\
\hline & & Target row & $F_{(2,14)}=617.46, P<0.001$ \\
\hline & & Target column & $F_{(2,14)}=13.89, \quad P<0.001$ \\
\hline & \multirow[t]{3}{*}{ Horizontal } & Orientation & $F_{(2,14)}=58.62, \quad P<0.001$ \\
\hline & & Target row & $F_{(2,14)}=53.37, \quad P<0.001$ \\
\hline & & Target column & $F_{(2,14)}=726.36, P<0.001$ \\
\hline \multirow[t]{9}{*}{ Lower arm } & \multirow{3}{*}{ Torsion } & Orientation & $F_{(2,14)}=225.74, P<0.001$ \\
\hline & & Target row & $F_{(2,14)}=0.83, \quad P>0.05$ \\
\hline & & Target column & $F_{(2,14)}=73.50, \quad P<0.001$ \\
\hline & \multirow[t]{3}{*}{ Vertical } & Orientation & $F_{(2,14)}=15.59, \quad P<0.001$ \\
\hline & & Target row & $F_{(2,14)}=838.24, P<0.001$ \\
\hline & & Target column & $F_{(2,14)}=21.65, \quad P<0.001$ \\
\hline & \multirow[t]{3}{*}{ Horizontal } & Orientation & $F_{(2,14)}=96.43, \quad P<0.001$ \\
\hline & & Target row & $F_{(2,14)}=27.90, \quad P<0.001$ \\
\hline & & Target column & $F_{(2,14)}=463.90, P<0.001$ \\
\hline \multirow{9}{*}{$\begin{array}{l}\text { Lower arm with } \\
\text { upper arm }\end{array}$} & \multirow[t]{3}{*}{ Torsion } & Orientation & $F_{(2,14)}=133.47, P<0.001$ \\
\hline & & Target row & $F_{(2,14)}=35.24, \quad P<0.001$ \\
\hline & & Target column & $F_{(2,14)}=31.52, \quad P<0.001$ \\
\hline & \multirow[t]{3}{*}{ Vertical } & Orientation & $F_{(2,14)}=2.47, \quad P>0.05$ \\
\hline & & Target row & $F_{(2,14)}=51.08, \quad P<0.001$ \\
\hline & & Target column & $F_{(2,14)}=27.14, \quad P<0.001$ \\
\hline & \multirow[t]{3}{*}{ Horizontal } & Orientation & $F_{(2,14)}=13.47, \quad P<0.001$ \\
\hline & & Target row & $F_{(2,14)}=105.91, P<0.001$ \\
\hline & & Target column & $F_{(2,14)}=10.30, \quad P<0.005$ \\
\hline
\end{tabular}

We thank S. Sun, T. Lew, and B, Deneau for technical support. We also thank the subjects for cooperation.

\section{I S C L O S UR E S}

This research was supported by grants from the Canadian Institutes of Health Research's Institute of Neurosciences, Mental Health and Addiction, and the McDonnell-Pew Program in Cognitive Neuroscience to J. J. Marotta, the Human Frontier Science Program to W.P. Medendorp, and the Natural Sciences and Engineering Research Council of Canada to J. D. Crawford.

\section{REFERENCES}

Andersen RA, Snyder LH, Batista AP, Buneo CA, and Cohen YE. Posterior parietal areas specialized for eye movements (LIP) and reach (PRR) using a common coordinate frame. In: Novartis Foundation Symposium, edited by Bock GR and Goode JA. New York: Wiley, 1998, p. 109-122.

Arbib M. Perceptual structures and distributed motor control. In: Motor Control. Handbook of Physiology, Section 1, The Nervous System, edited by Brooks V. Baltimore: Williams and Wilkins, 1981, vol. 2 p. 1449-1480.

Arbib M. Programs, schemas, and neural networks for control of hand movements: beyond the RS framework. In: Attention and Performance, edited by Jeannerod M. Hillsdale, NJ: Erlbaum, 1990, p. 111-138.

Arbib M, Iberall T, and Lyons D. Coordinated control programs for movements of the hand. Exp Brain Res 10: 111-129, 1985.

Batista AP, Buneo CA, Snyder LH, and Andersen RA. Reach plans in eye-centered coordinates. Science 285: 257-260, 1999.

Caminiti R, Ferraina S, and Johnson PB. The sources of visual information to the primate frontal lobe: a novel role for the superior parietal lobule. Cereb Cortex 6: 319-328, 1996.

Ceylan M, Henriques DY, Tweed DB, and Crawford JD. Task-dependent constraints in motor control: pinhole goggles make the head move like an eye. J Neurosci 20: 2719-2730, 2000.

Connolly JD, Goodale MA, Desouza JFX, Menon RS, and Vilis T. A comparison of frontoparietal fMRI activation during anti-saccades and antipointing. J Neurophysiol 84: 1645-1655, 2000.

Crawford JD, Ceylan MZ, Klier EM, and Guitton D. Three-dimensional eye-head coordination during gaze saccades in the primate. J Neurophysiol 81: 1760-1782, 1999.

Crawford JD and Vilis T. How do motor systems deal with the problem of controlling three-dimensional rotations? J Motor Behav 27: 89-99, 1995. 
Desmurget M, Gréa H, and Prablanc C. Final posture of the upper limb depends on the initial position of the hand during prehension movements. Exp Brain Res 119: 511-516, 1998.

Desmurget M, Prablanc C, Arzi M, Rossetti Y, Paulignan Y, and Urquizar C. Integrated control of hand transport and orientation during prehension movements. Exp Brain Res 110: 265-278, 1996.

Fitts $\mathbf{P}$. The information capacity of the human motor system in controlling the amplitude of movement. J Exp Psychol 47: 381-391, 1954.

Flanders M, Hondzinski J, Soechting J, and Jackson J. Using arm configurations to learn the effects of gyroscopes and other devices. J Neurophysiol 89: 450-459, 2003

Gallese V, Murata A, Kaseda M, Niki N, and Sakata H. Deficit of hand preshaping after muscimol injection in monkey parietal cortex. Neuroreport 5: $1525-1529,1994$

Gentilucci M, Castiello U, Corradini ML, Scarpa M, Umilta C, and Rizzolatti G. Influence of different types of grasping on the transport component of prehension movements. Neuropsychologia 29: 361-378, 1991.

Gielen CC, Vrijenhoek EJ, Flash T, and Neggers SF. Arm position constraints during pointing and reaching in 3-D space. J Neurophysiol 78 660-673, 1997

Glenn B and Vilis T. Violations of Listing's law after large eye and head gaze shifts. J Neurophysiol 68: 309-318, 1992.

Haustein W. Considerations on Listing's Law and the primary position by means of a matrix description of eye position control. Biol Cybern 60 411-420, 1989

Hepp K, Haslwanter T, Straumann D, Hepp-Reymond M-C, and Henn V. The control of arm-, gaze-, and head-movements by Listing's law. In Control of Arm Movements in Space: Neurophysiological and Computational Approaches, edited by Caminiti R, Johnson P, and Burnod Y. Berlin Heidelberg New York: Springer, 1992, p. 307-319.

Hore J, Watts S, and Vilis T. Constraints on arm position when pointing in three dimensions: Donders' law and the Fick gimbal strategy. J Neurophysiol 68: 374-383, 1992.

Jeannerod M. Intersegmental coordination during reaching at natural visual objects. In: Attention and Performance, edited by Long J and Baddeley A. Hillsdale: Erlbaum, 1981, p. 153-168.

Jeannerod M. The timing of natural prehension movements. J Motor Behav 16: 235-254, 1984.

Jeannerod M. The Neural and Behavioural Organization of Goal-directed Movements. Oxford: Clarendon, 1988.

Jeannerod M. Coordination mechanisms in prehension movements. In: $T u-$ torials in Motor Behavior II, edited by Stelmach G and Requin J. Amsterdam: Elsevier Science, 1992, p. 265-285.

Jeannerod $M$ and Biguer B. Visuomotor mechansims in reaching within extrapersonnal space. In: Analysis of Visual Behavior, edited by Ingle D Goodale M, and Mansfield R. Cambridge: MIT Press, 1982, p. 387-409.

Jeannerod $\mathbf{M}$ and Decety $\mathbf{J}$. The accuracy of visuomotor transformation: an investigation into the mechanisms of visual recognition of objects. In: Vision and Action: The Control of Grasping, edited by Goodale MA. Westport, CT: Ablex Publishing, 1990, p. 33-48.

Lacquaniti $\mathbf{F}$ and Soechting JF. Coordination of arm and wrist motion during a reaching task. J Neurosci 2: 399-408, 1982.

Mamassian P. Prehension of objects oriented in three-dimensional space. Exp Brain Res 114: 235-245, 1997.
Martinez-Trujillo J, Wang H, and Crawford J. Electrical stimulation of the supplementary eye fields in the head-free macaque evokes kinematically normal gaze shifts. J Neurophysiol 89: 2961-2974, 2003.

Medendorp WP, Crawford JD, Henriques DY, Van Gisbergen JA, and Gielen CC. Kinematic strategies for upper arm-forearm coordination in three dimensions. J Neurophysiol 84: 2302-2316, 2000.

Miller $\mathbf{L}$, Theeuwen $\mathbf{M}$, and Gielen $\mathbf{C}$. The control of arm pointing movements in three dimensions. Exp Brain Res 90: 415-426, 1992.

Murata A, Gallese V, Kaseda M, and Sakata H. Parietal neurons related to memory-guided hand manipulation. J Neurophysiol 75: 2180-2186, 1996.

Murata A, Gallese V, Luppino G, Kaseda M, and Sakata H. Selectivity for the shape, size, and orientation of objects for grasping in neurons of monkey parietal area AIP. J Neurophysiol 83: 2580-2601, 2000.

Oldfield RC. The assessment and analysis of handedness: the Edinburgh inventory. Neuropsychologia 9: 97-113, 1971.

Paulignan Y, Frak V, Toni I, and Jeannerod M. Influence of object position and size on human prehension movements. Exp Brain Res 114: 226-234, 1997.

Paulignan Y, Jeannerod M, MacKenzie C, and Marteniuk R. Selective perturbation of visual input during prehension movements. 2. The effects of changing object size. Exp Brain Res 87: 407-420, 1991a.

Paulignan Y, MacKenzie C, Marteniuk R, and Jeannerod M. Selective perturbation of visual input during prehension movements. 1. The effects of changing object position. Exp Brain Res 83: 502-512, 1991b.

Sakata H, Taira M, Murata A, Gallese V, Tanaka Y, Shirikata E, and Kusunoki M. Parietal visual neurons coding three-dimensional characteristics of objects and their relation to hand action. In: Parietal Lobe Contributions to Orientation in 3D Space. Experimental Brain Research, edited by Thier P and Karnath H. Heidelberg: Springer-Verlag, 1997, p. 237-254.

Smeets JB and Brenner E. A new view on grasping. Motor Control 3: 237-271, 1999.

Snyder LH, Batista AP, and Andersen RA. Intention-related activity in the posterior parietal cortex: a review. Vision Res 40: 1433-1441, 2000.

Soechting JF, Buneo CA, Herrmann U, and Flanders M. Moving effortlessly in three dimensions: does Donders' law apply to arm movement? J Neurosci 15: 6271-6280, 1995.

Soechting JF and Flanders M. Parallel, interdependent channels for location and orientation in sensorimotor transformations for reaching and grasping. J Neurophysiol 70: 1137-1150, 1993.

Stelmach G, Castiello U, and Jeannerod M. Orienting the finger opposition space during prehension movements. J Motor Behav 26: 178-186, 1994.

Straumann D, Haslwanter T, Hepp-Reymond MC, and Hepp K. Listing's law for eye, head and arm movements and their synergistic control. Exp Brain Res 86, 209-215, 1991

Taira M, Mine S, Georgopoulos AP, Murata A, and Sakata H. Parietal cortex neurons of the monkey related to the visual guidance of hand movement. Exp Brain Res 83: 29-36, 1990.

Theeuwen M, Miller LE, and Gielen CC. Are the orientations of the head and arm related during pointing movements? J Motor Behav 25: 242-250, 1993.

Tillery SI, Ebner TJ, and Soechting JF. Task dependence of primate arm postures. Exp Brain Res 104: 1-11, 1995.

Wallace S and Weeks D. Temporal constraints in the control of prehensile movement. J Motor Behav 20: 81-105, 1988. 